\title{
Indoor Propagation Model for TV White Space
}

\author{
Wataru Yamada, Motoharu Sasaki \\ Takatoshi Sugiyama, \\ NTT Access Network Services Systems Laboratories \\ NTT Corporation \\ Yokosuka, Japan
}

\author{
Oliver Holland, Shuyu Ping, Bright Yeboah-Akowuah, \\ Jiwon Hwang, Hamid Aghvami \\ Centre for Telecommunications Research \\ King's College London \\ London, United Kingdom
}

\begin{abstract}
In this paper, indoor propagation characteristics for TV white space (TVWS) frequency bands are studied. Measurements in the actual indoor environment are performed and described. An indoor propagation model for TVWS is developed, which is useful for evaluating secondary-secondary interference in TVWS scenarios, and its empirical parameters are derived according to measurement results. Our proposed model is based on components of free space path loss, penetration losses of walls and floors, an attenuation coefficient against distance, and an attenuation constant. Frequency dependency of these parameters is derived. Our proposed model is compared with representative indoor models from the literature. Results show that our proposed model, with the obtained empirical parameters, achieves better performance than representative indoor models. The standard deviation of estimation error for our proposed model is only $5.9 \mathrm{~dB}$.
\end{abstract}

Keywords-Frequency sharing systems; TVWS; Propagation measurement; Indoor propagation model; Interference evaluation;

\section{INTRODUCTION}

Data traffic demand is increasing year by year [1], and there is a huge need for increased spectrum for wireless communication systems to satisfy this demand. However, there is little or no extra availability in any of the spectrum bands appropriate for wireless communication services. Spectrum sharing is one of the means to address this issue [2]. Particularly, spectrum sharing between television broadcast and other communication systems is possible because many channels in spectrum allocated to television services are not being used in many locations [3].

Systems in licensed bands are generally categorized as primary or secondary [4], whereby in television bands the primary systems are dominantly television broadcast or wireless microphone systems. Secondary systems must not interfere with these primary systems. Further, there are many different types of secondary systems being standardized for operation in TVWS, and interference among all of these systems is a concern regarding the expectations on their performance as experienced by the end-user, especially in locations where the number of available channels for secondary usage is limited.

To evaluate spectrum sharing, interference between systems should be assessed [5]. An understanding of propagation characteristics is TVWS, in scenarios pertinent to secondarysecondary geometries, is an essential factor in better predicting interference in secondary coexistence. Transmitters and receivers of different secondary systems and links might reside indoor in nearby locations [6]. Therefore, it is necessary to apply propagation models for indoor-to-indoor scenarios to assess secondary-secondary coexistence.

Many propagation models for TV bands have been proposed in the literature (see, e.g., [7], [8]), and numerous evaluations of interference characteristics from secondary systems to primary systems have been performed (e.g., [9]). However, these propagation models are mostly based on TV broadcasting, and are generally for long distances in outdoor scenarios. Few propagation studies for short distance transmission have been reported for TV bands, and particularly, very few studies on indoor propagation have been done.

In view of the above, this paper performs propagation measurements in an actual indoor environment. Seven frequencies are assessed, ranging from $471 \mathrm{MHz}$ to $775 \mathrm{MHz}$. An indoor propagation model for TV bands is developed based on the measurement results. This paper is structured as follows. Section II introduces the measurements performed by us, whereby Section III introduces our propagation modeling methodology and the resulting model. Section IV shows measurement results and estimation results, before Section V introduces frequency dependency in the model and further validates that. Finally, Section VI concludes.

\section{MEASUREMENT ENVIRONMENT AND PARAMETERS}

Our measurements were carried out in the Strand building of King's College London's Strand Campus. The measurement equipment configuration was as shown in Figure 1. Here, SG is signal generator, ATT is attenuator, and HPA is high power amplifier.

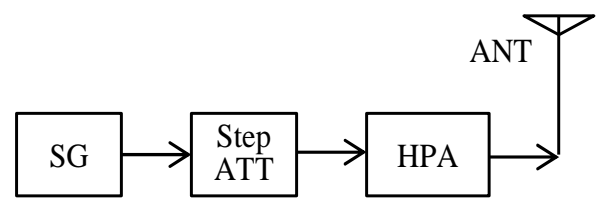

(a) transmitter

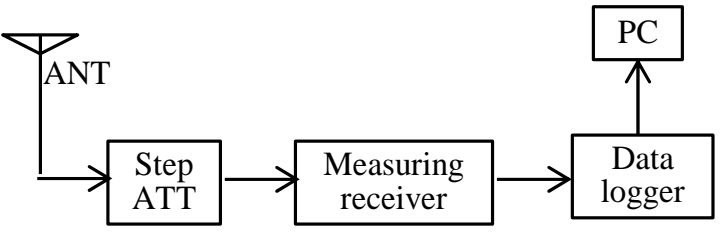

(b) receiver

Figure 1. Measurements equipment configuration 
In this building, the second to fourth floor were measured. Fig. 2(a)-(c) show the deployed layout of the environment, and Table I summarizes measurement parameters.

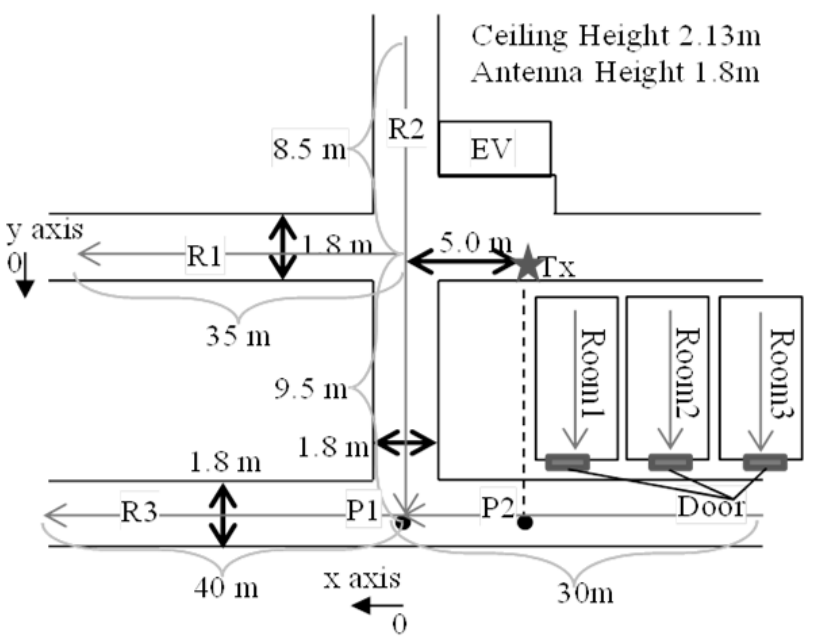

Figure 2(a). Floor plan of second floor

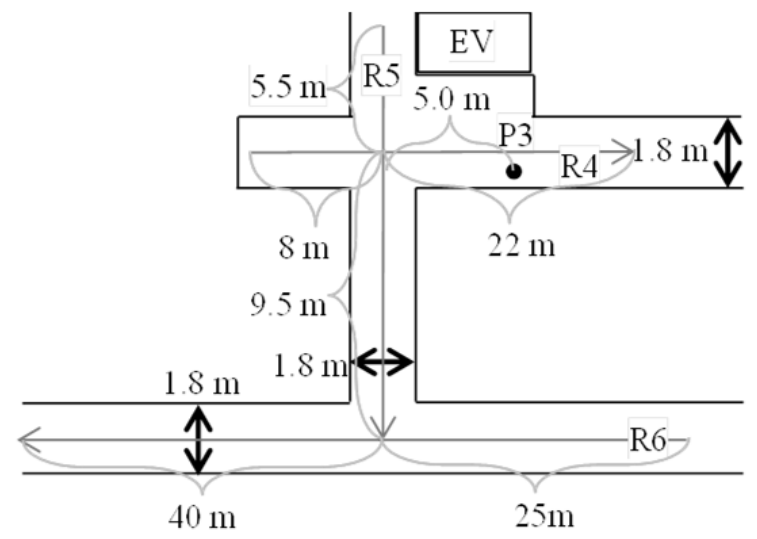

Figure 2(b). Floor plan of third floor

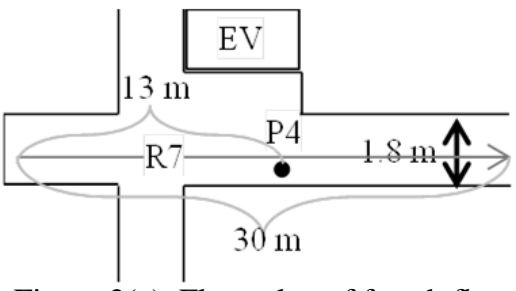

Figure 2(c). Floor plan of fourth floor

The transmitter was set on the second floor, as shown in Figure 2(a). The antenna height was $1.8 \mathrm{~m}$. The receiver and data logger were placed on a trolley and moved at a walking pace of approximately $0.5 \mathrm{~m} / \mathrm{s}$ along the line of the predetermined route. The receive antenna height was also set to $1.8 \mathrm{~m}$ to eliminate the effect of human body attenuation. The data sampling rate of the data logger was $1.5 \mathrm{kHz}$, and the resolution bandwidth was set to $15 \mathrm{kHz}$. The antenna radiation pattern was omni-directional in the horizontal plane for both transmitter and receiver. In United Kingdom, TVWS frequency bands are defined between $470 \mathrm{MHz}$ to $790 \mathrm{MHz}$ [4]. Seven transmission frequencies were used covering this range in approximately equal steps, including frequencies close to its extremes. A continuous wave signal was transmitted at each frequency.

Table I. Measurement Parameters

\begin{tabular}{|l|l|}
\hline $\begin{array}{l}\text { Measured frequencies } \\
\text { (MHz) }\end{array}$ & $\begin{array}{l}\text { 471.6625, 521.15, 570, } \\
\text { and 775 (CW) }\end{array}$ \\
\hline $\begin{array}{l}\text { Antenna radiation } \\
\text { pattern }\end{array}$ & $\begin{array}{l}\text { Omni-directional in } \\
\text { horizontal plane }\end{array}$ \\
\hline Antenna Gain & $0 \mathrm{dBi}$ \\
\hline Height of antenna & $1.8 \mathrm{~m}$ for both Tx and Rx \\
\hline Data sampling rate & $1.5 \mathrm{kHz}$ \\
\hline Resolution bandwidth & $15 \mathrm{kHz}$ \\
\hline Dynamic range & over 70 dB \\
\hline
\end{tabular}

On the second floor, three routes in the corridor were chosen: R1 (route for Line-of-Sight conditions), R2 (route with propagation around one corner), and R3 (route with propagation around two corners). Three routes in the rooms were chosen. On the third floor, three routes in the corridor, R4 to R6 as shown in Figure 2(b), were chosen for measurements. Point P3 is directly above the transmitter on the second floor, and these routes are located just above the routes on the second floor. On the fourth floor, one route, R7, was chosen for measurements. Point P4 is directly above the transmitter on the second floor and R7 is located directly above R1and R4.

\section{Modelling OF INDOOR PROPAGATION CHANNEL IN TVWS FREQUENCY BANDS}

Generally, two main options for propagation paths are considered in indoor propagation modeling. The first one is the path following open space, and the other is the direct path, including penetration through walls and other obstacles [10]. In the case of an office building, the path following open space generally corresponds to the path along the corridors, and the direct path generally corresponds to the path crossing through rooms, as shown in Route A1 and A2 respectively, Figure 3.

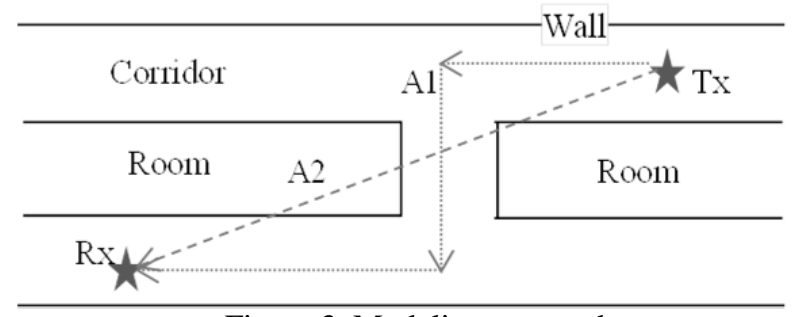

Figure 3. Modeling approach

Figure 4 shows the measurement results for Route R3 at $609 \mathrm{MHz}$. In this Figure, the estimation calculated by double knife edge model is also plotted. Here, measurement results are averaged over $0.1 \mathrm{~m}$ sections to mitigate the effects of fast fading, and that same processing method is applied hereafter. Looking at this Figure, the estimation result calculated by double knife edge model are far from the measurement results. It is also shown that the propagation loss on the negative region 
of the $x$-axis less than that of the positive region. Propagation loss around position P1 would be expected to minimize propagation loss if the propagation path along the corridor was the dominant path. However, it is shown the propagation loss around point $\mathrm{P} 2$ is the least. This indicates the dominant path in an indoor environment in the TVWS frequency band is the direct path. Therefore, the indoor propagation model in TVWS can be described as a function of the direct distance between the transmitter and receiver.

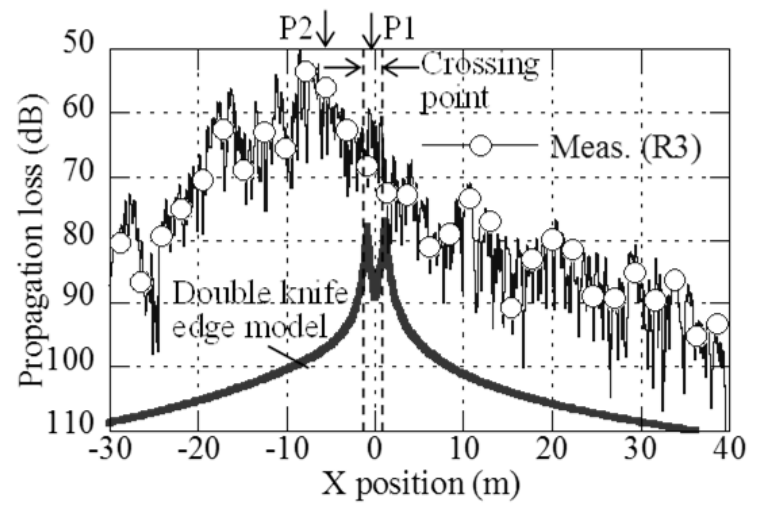

Figure 4. Measurement results for R3 and comparison with the double knife-edge model

Figure 5 shows the propagation loss characteristics for Route R3 on the second floor and R6 on the third floor in the $609 \mathrm{MHz}$ band. In this Figure, similarly to the results for the second floor, we observe a propagation loss at negative $\mathrm{X}$ position locations of less than the positive locations on the third floor. Moreover, we find that the difference between these propagation characteristics is almost constant.

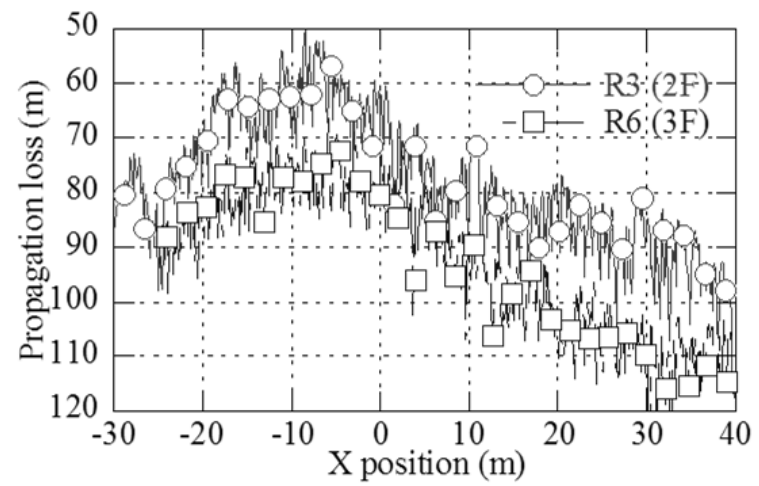

Figure 5. Measurement results for Routes R3 and R6

Up to now, many indoor propagation models based on direct distance have been proposed. Representative such models are the COST 231 model [11], and the ITU-R P.1238 model [12]. In the document describing COST 231, two models are proposed. One is a multi-wall model (MWM) and the other is a linear attenuation model (LAM). These models are formulated as

$$
\begin{aligned}
& \text { MWM: } L=L_{F S}+L_{C}+\sum_{i=1}^{l} k_{w i} L_{w i}+k_{f}\left[\frac{k_{f}+2}{k_{f}+1}-b\right] L_{f}, \\
& \text { LAM: } L=L_{F S}+\alpha d, \\
& \text { ITU-R model: } L=\operatorname{20log}_{0}(f)+N \log _{0}(d)+L_{f}\left(n_{f}\right)-28,
\end{aligned}
$$

where $L$ is the propagation loss, $L_{F S}$ is the free-space propagation loss between the transmitter and receiver, $L_{c}$ is a constant loss, $k_{w i}$ is the number of penetrated walls of type $i, L_{w i}$ is loss of each wall of type $i, k_{f}$ is the number of penetrated floors, $L_{f}$ is the loss between adjacent floors, $b$ is the empirical parameter, $l$ is the number of wall types, $\alpha$ is the attenuation coefficient, $d$ is the distance between the transmitter and receiver in meters, $f$ is the frequency in $\mathrm{MHz}, N$ is the distance power-loss coefficient, and $n_{f}$ is the number of penetrated walls. These equations are primarily developed for frequencies of over $800 \mathrm{MHz}$, so our target frequency band is out of range. However, we have performed calculations of these equations for Route R3 as a justification. Parameters for the calculations are $L_{w 2}=6.9$ for MWM, $\alpha=0.62$ for LAM, and $N=33$ for ITU-R P.1238 model. Note that a door was not considered as a wall in this paper, because most of the door is made of glass and glass has only a slight penetration loss. These results are shown in Figure 6

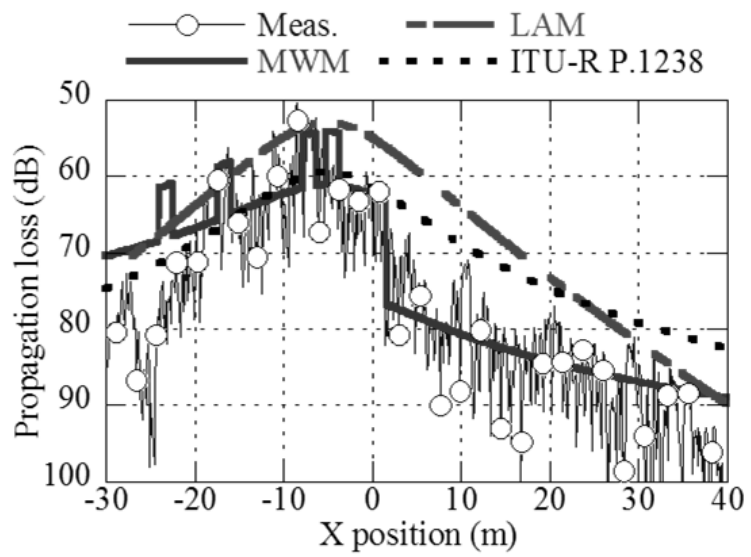

Figure 6. Measurement and estimation results

In this Figure, both the LAM and ITU-R P.1238 models have large errors, especially in the positive $\mathrm{X}$-position region. These errors are up to $10 \mathrm{~dB}$. On the other hand, MWM shows a similar tendency to measurement results. Therefore, this model looks similar at the first glance. However, with careful attention, it is found that the estimation error gradually increases as distance increases-this is because the gradient against distance is smaller than the measurement results. Therefore, MWM with a term added representing an additional decay against distance seems to be an appropriate model for this frequency band.

Generally, space, walls, ceilings or floor boards, and some obstacles, such as furniture, are the elements between the transmitter and receiver in an indoor environment. It is necessary to consider these obstacles to model propagation 
since each obstacle has specific attenuation coefficients and this influences to propagation characteristics. However, it is difficult to know where these obstacles are placed inside rooms. Therefore, we assume obstacles are uniformly distributed in the space and have a uniform attenuation coefficient. Using this assumption, the following equation can be derived,

$$
L=L_{F S}+\alpha d+n_{w} L_{w}+n_{f} L_{f}+C,
$$

where $n_{w}$ is number of penetrating walls and $C$ is attenuation constant. In this paper, we have defined this equation as our indoor propagation model for TVWS frequencies, and have analyzed measured propagation data based on this equation. This model can be considered as a variation on existing models. However, some parts are abbreviated by comparing this model with existing models. Based on our measurement results, this simplification of propagation model is effective. These will be shown in the next section.

\section{MEASUREMENT RESULTS AND EMPRICAL PARAMETERS}

\section{A. Line-of-Sight Condition}

Based on the propagation model defined in the previous section, we have derived empirical parameters for $\alpha$ and $C_{L O S}$ by using the measurement results for the Line-of-Sight situation on the second floor. This corresponds to Route R1. These parameters are calculated based on the least square fitting technique. Figure 7 shows the result of measurement, as straight line with circle, and an approximation, shown as a straight line. Calculated results for free space path loss, shown as a dotted line, are also plotted in this Figure.

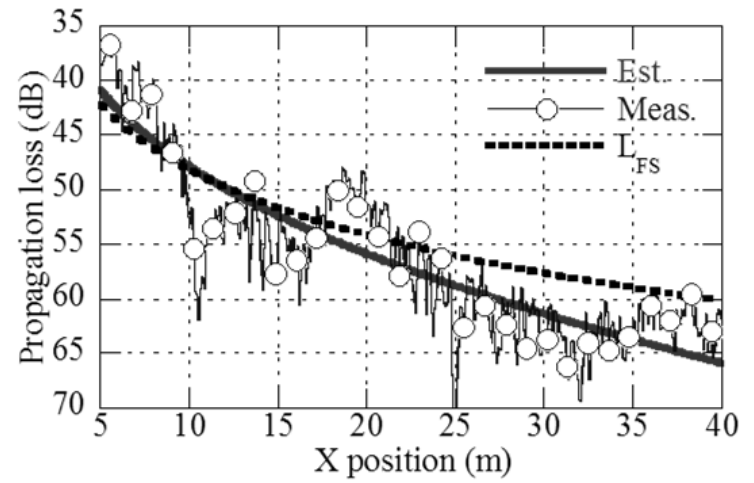

Figure 7. Measurement and estimation results (609 MHz)

Here, the horizontal axis represents the position of the receiver, where the defined position is shown in Figure 2(a). From this Figure, measurement results indicate a slightly higher propagation loss than free space loss. This is caused by the effects of reflection waves from walls. On the other hand, estimation results based on our model can accurately predict measurement data. In this case, derived parameters in this 609 $\mathrm{MHz}$ band were 0.17 for $\alpha \mathrm{dB} / \mathrm{m}$ and -1.7 for $C_{L O S} \mathrm{~dB}$, respectively.

\section{B. Non-Line-of Sight Condition}

Empirical parameters for $L_{w}$ and $C_{N L O S}$ by using the measurement result for the Non-Line-of-Sight situation on the second floor are derived. Here, the value for $\alpha$ was adopted as derived in the previous section. Parameters were derived from two routes in the corridors and three routes in the rooms, also using the least squares fitting technique. Note that we defined walls as those next to corridors. It was relatively easy to be sure of the characteristics of the walls next to corridors whereas it was more difficult for those walls dividing rooms. This is because it's often the case that a corridor is a common space and a room is a private space. Moreover, there are unpredictable elements such as cabinets and partitions inside or dividing rooms, and such walls between rooms tend to be generally relatively flimsy. Given this, we didn't take into account walls separating rooms-this is also convenient for practical usage. In this case, derived parameters at $609 \mathrm{MHz}$ were 5.9 for $L_{w}$ in $\mathrm{dB}$, and 1.4 for $C_{N L O S}$ in $\mathrm{dB}$, respectively.

Figure 8 gives the results of measurement and approximation on Route R2. The distance between $-2 \mathrm{~m}$ and 1 $\mathrm{m}$ is line-of-sight, whereas other distances are non-line-of-sight.

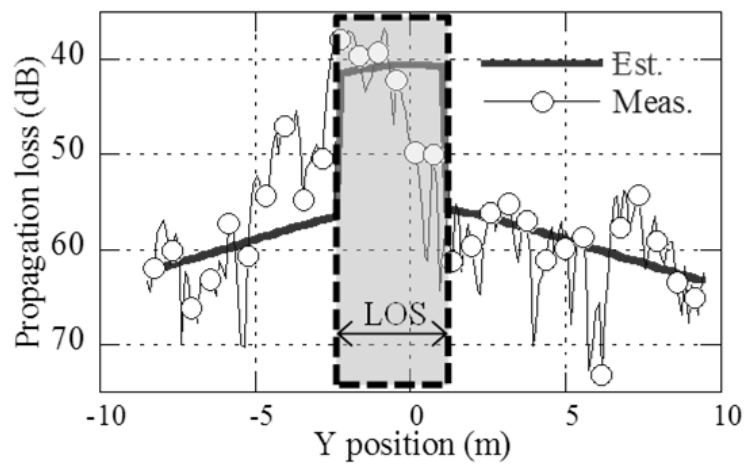

Figure 8. Results for Route R2 (609 MHz)

The measured path loss is lower than the estimated loss between $-2 \mathrm{~m}$ to $-5 \mathrm{~m}$. This is because there is a single reflection of the transmitted wave in that region. In the other regions, estimation results show a good performance.

Next, the results of measurements and the approximation on Route R3 is shown in Figure 9.

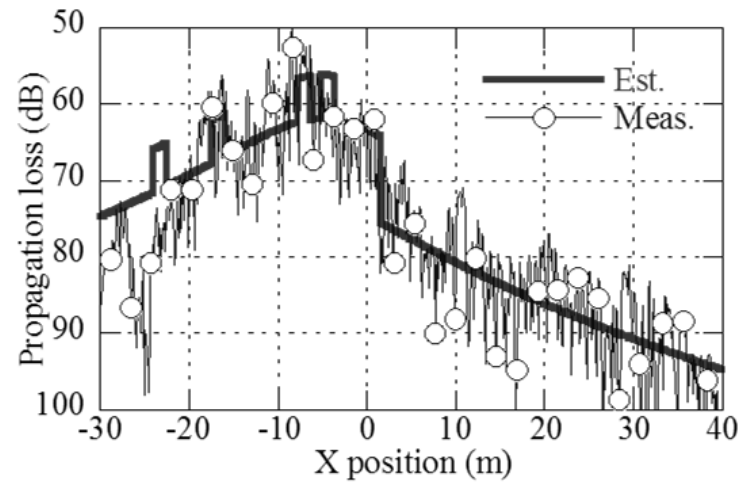

Figure 9. Results for Route R3 (609 MHz)

Around the +1 meters position, the measurement results indicate a large shift in propagation loss. This can also be seen 
in the estimation results. This area corresponds to the changing point of the number of walls from two to four. Further, estimated propagation loss is decreased at some minus position. This area has only one wall due to the existence of a door (see Figure 2(a)). On the other hand, similarly to the estimation results, measurement results show a decreased propagation loss at some negative position. In the other regions, the estimation results show a good performance. In this way, the change of propagation loss can emulated by considering the number of walls between the transmitter and receiver that are introduced in our proposed model.

Figure 10 gives the results of measurements and approximation in room 1 to 3 . Note that horizontal axis is changed to direct distance between the transmitter and receiver.

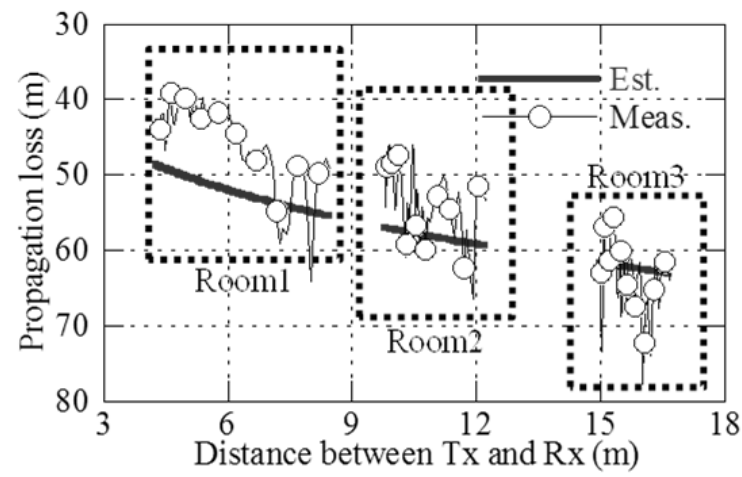

Figure 10. Result of Route in room 1 to 3 (609 MHz)

In this Figure, an estimation error of about $5 \mathrm{~dB}$ in room 1 and $3 \mathrm{~dB}$ in room 2 is observed. On the other hand, estimation results in room 3 show a good performance.

\section{Floor penetration loss}

Next, empirical parameters for $L_{f}$ are derived. The values for $\alpha, L_{w}$ and $C_{N L O S}$ were adopted as derived in the previous sections. These parameters were derived from four routes, in the corridors of the third and fourth floor compared with that of the second floor. In this case, the derived empirical parameter was $14.0 \mathrm{~dB}$ for $L_{f}$ at $609 \mathrm{MHz}$. Figure 11 gives the results of measurements and approximations on Routes R3 and R6.

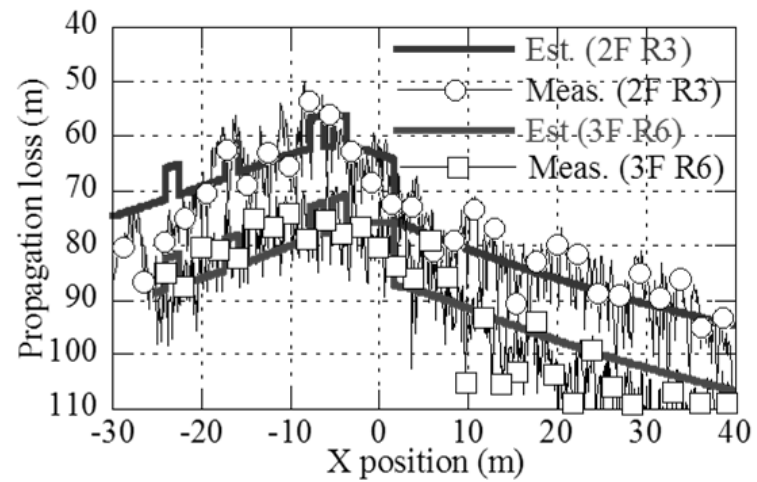

Figure 11. Floor penetration loss results on Routes R3 and R6 (609 MHz)
In this Figure, estimation results on Route R6 show a good performance. Although we spare showing results for the other route in this paper because space limitations, we can confirm the results on the other route have achieve a good performance.

\section{PRoposed INDOOR PROPAGATION MOdEL WITH FREQUENCY DEPENDENT PARAMETERS}

In the previous section, it is shown that our propagation model can be a reliable model in terms of accuracy. Here, frequency dependant parameters are examined using all the measured data for all of the measurement frequencies. Figure 12 shows the results of wall penetration loss $L_{w}$ and floor penetration loss $L_{f}$ for all the measured frequencies.

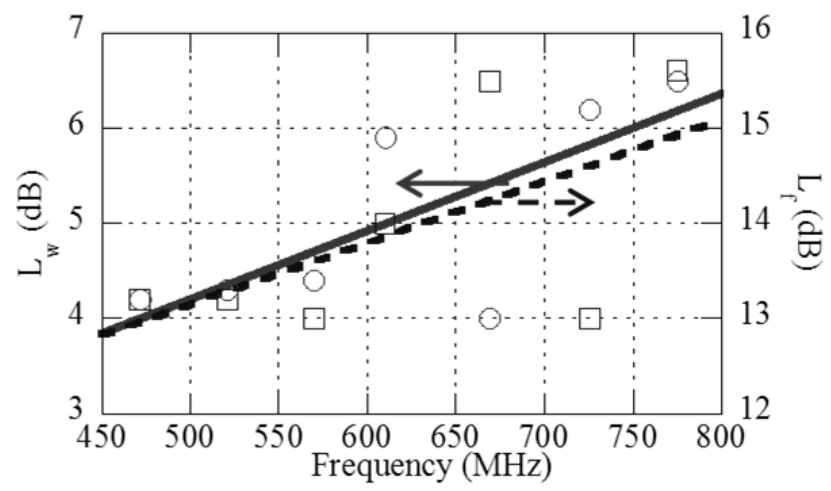

Figure 12. Frequency characteristics for $L_{w}$ and $L_{f}$

The range of $L_{w}$ is around $4 \mathrm{~dB}$ to $7 \mathrm{~dB}$ for the measured data. Further, the range of $L_{f}$ is around $14 \mathrm{~dB}$ to $20 \mathrm{~dB}$ for the measured data. Both values increase with increasing frequency. From the tendencies of the measured data, approximation curves are derived as follows:

$$
\begin{aligned}
L_{w} & =0.60+7.2 \times 10^{-3} f, \\
L_{f} & =9.9+6.5 \times 10^{-3} f .
\end{aligned}
$$

Figure 13 gives the results for the attenuation coefficient $\alpha$ and attenuation constant $C$ for all the measured frequencies.

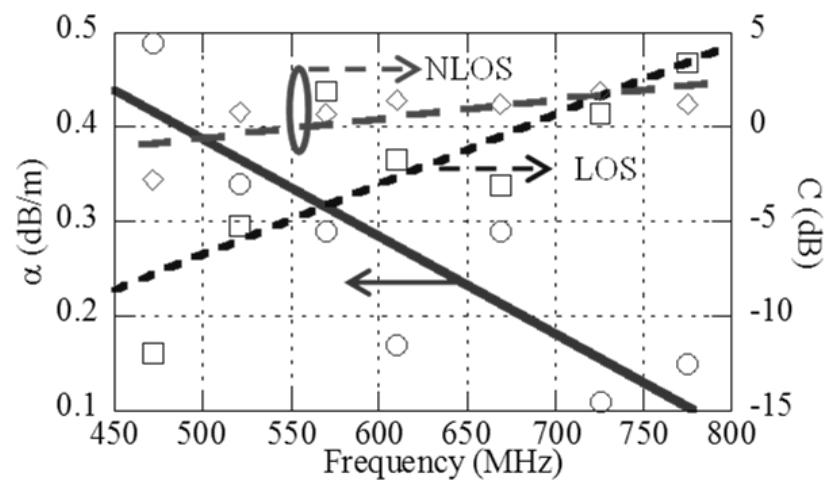

Figure 13. Frequency dependency for $\alpha$ and $C$

The range of $C_{L O S}$ is around $-3 \mathrm{~dB}$ to $2 \mathrm{~dB}$ and $C_{N L O S}$ is around $-12 \mathrm{~dB}$ to $4 \mathrm{~dB}$ for the measured data. Both values 
increase with increasing frequency. On the other hand, the range of $\alpha$ is around $0.1 \mathrm{~dB}$ to $0.5 \mathrm{~dB}$ for the measured data. Values for $\alpha$ decrease with increasing frequency. From the tendencies of the measured data, approximation curves are derived as

$$
\begin{gathered}
\alpha=0.90-1.0 \times 10^{-3} f, \\
C_{L O S}=-25+3.7 \times 10^{-2} f, \\
C_{N L O S}=-5.7+1.0 \times 10^{-2} f .
\end{gathered}
$$

Finally, the proposed model using equations (5) to (8), has been compared with the MWM, LAM, ITU-R P.1238 models in terms of estimation error. Figure 14 shows the cumulative probability distribution of the estimation error. The population is including all the data for the second, third and fourth floors. A positive estimation error means that the estimated path loss is less than the measured path loss. Here, the standard deviation of the error is $5.9 \mathrm{~dB}$ for the proposed model, $7.0 \mathrm{~dB}$ for MWM, $16.2 \mathrm{~dB}$ for LAM and $11.3 \mathrm{~dB}$ for the ITU-R P.1238 model.

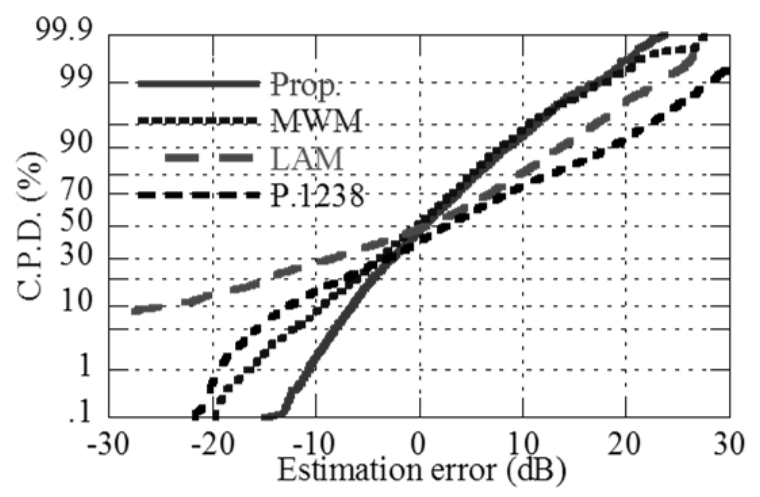

Figure 14. Estimation error analysis (609 MHz)

\section{CONCLUSION}

Frequency sharing is a possibility to address the demands of growing data traffic. To evaluate frequency sharing systems, propagation modeling is required. So far, there are few studies for indoor propagation modeling that can apply to TVWS.

In this paper, indoor propagation characteristics for TVWS frequency bands were studied. Measurements in the actual indoor environment were performed. Next, an indoor propagation model for TVWS frequency bands to evaluate interference power and empirical parameters was derived according to the measurement results. This proposed model was based on the free space path loss, penetration losses of walls and floors, an attenuation coefficient and attenuation constant. Frequency dependency of these parameters was derived, and the proposed model was compared with representative indoor models. As a result, it was shown that the proposed model achieves better performance than representative indoor models and the standard deviation of estimation error is $5.9 \mathrm{~dB}$. Finally, it should be noted that this model not only evaluates interference power, but also can be used for coverage provisioning.

\section{ACKNOWLEDGMENT}

The authors wish to thank Paul Pangalos and Idongesit Udoessien for their helpful support towards our propagation measurement campaign.

\section{REFERENCES}

[1] "Cisco Visual networking Index: Global Mobile Data Traffic Forecast U pdate, 2012-2017,“ http://www.cisco.com/en/US/solutions/collateral/ns3 41/ns525/ns537/ns705/ns827/white_paper_c11-520862.pdf, Feb. 2013.

[2] R. Q. Hu, Y. Qian, S. Kota, and G. Gimbene, "HetNets-A new paradigm for increasing cellular capacity and coverage,” IEEE Wireless Commun. Mag., vol. 18, no. 3, pp. 8-9, June 2011.

[3] Ofcom, "Regulatory Requirements for White Space Devices in the UHF TV Band,” http://www.cept.org/Documents/se-43/6161/, July 4, 2012.

[4] Ofcom, “TV white spaces: approach to coexistence," 04 Sep. 2013, http://stakeholders.ofcom.org.uk/binaries/consultations/white-spacecoexistence/summary/white-spaces.pdf.

[5] F. Letourneux, S. Guivarch, and Y. Lostanlen, "Propagation models for Heterogeneous Networks," Trans. on 7th European Conference on Antenna and Propagation, pp.3993-3997, April 2013.

[6] S. Kawade, and M. Nekovee, "Can Cognitive Radio Access to TV White Spaces Support Future Home Networks?,” Trans. on 2010 IEEE Symp. On New Frontiers in Dynamic Spectrum, pp. 1-8, April 2010.

[7] M. Hata, "Empirical formula for propagation loss in land mobile radio services,” IEEE Trans. Veh. Tech., vol. 29, No. 3, pp. 317-325, 1980.

[8] A. G. Longley and P. L. Rice, "Prediction of tropospheric radio transmittion loss over irregular terrain,” U.S. Department of Commerce, ESSA Rep. ERI-79-ITS-67, 1968.

[9] G. P. Villardi, Y. D. Alemseged, C. Sun, C.-S. Sum, T. H. Nguyen, T. Baykas, and H. Harada, "Enabling coexistene of multiple cognitive networks in TV white space,” IEEE Wireless Commun. Mag., August 2011.

[10] X. Zhao, S. Geng, and B. M. Coulibaly, "Path-Loss Model Including LOS-NLOS Transition Regions for Indoor Corridor at $5 \mathrm{GHz}$," IEEEAntennas and Propagation Magazine, vol 33, no. 3, pp.217-223, June, 2013.

[11] "Digital Mobile Radio: COST 231 View on the Evolution Towards 3rd Generation Systems," Commission of the European Communities, L2920, Luxembourg, 1989.

[12] Propagation data \& prediction method for the planning of indoor radio communication systems and radio local area networks in the frequency range $900 \mathrm{MHz}$ to $100 \mathrm{GHz}$, ITU-R P.1238-7, 2012. 